$\begin{array}{ll}\text { Research Square } & \text { Preprints are preliminary reports that have not undergone peer review. } \\ \text { They should not be considered conclusive, used to inform clinical practice, } \\ \text { or referenced by the media as validated information. }\end{array}$

\title{
An Assessment of Implementation of Mass Drug Administration for Prevention and Control of Schistosomiasis and Soil-Transmitted Helminths in Selected Southern Malawi Districts
}

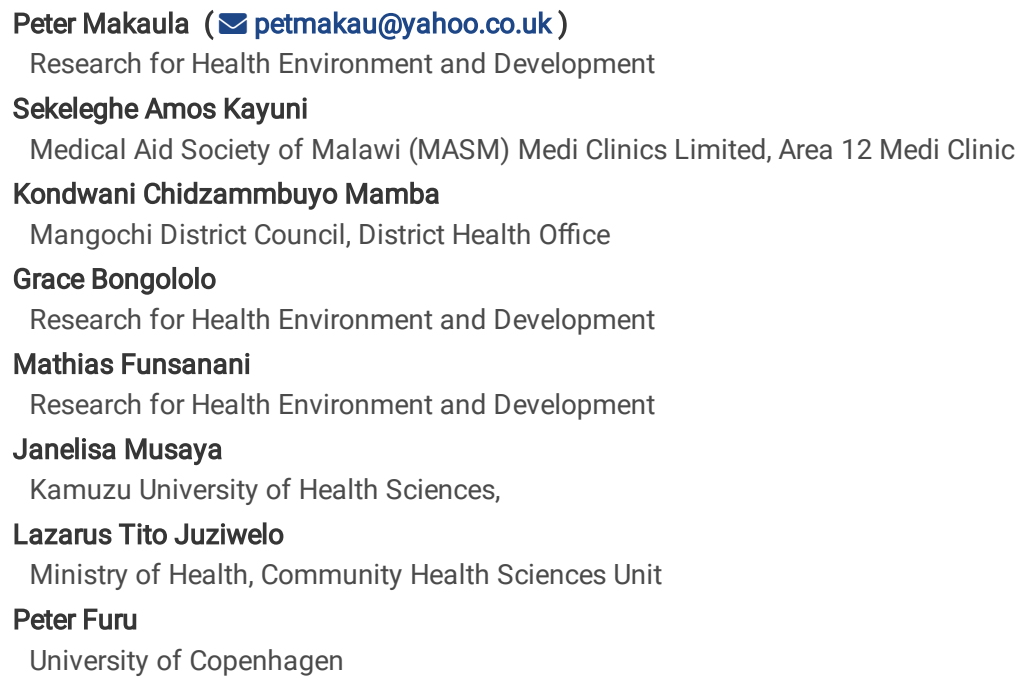




\section{Abstract \\ Background}

Mass drug administration (MDA) is one of the key interventions recommended by WHO for prevention and control of neglected tropical diseases. In Malawi, MDA is widely carried out annually since 2012 for prevention and control of schistosomiasis and soil transmitted helminths (STH). No study has been carried out to assess effectiveness of the approach and to document perceptions of health providers and beneficiaries regarding use of MDA. This study aimed to examine perceived strengths and weaknesses, successes and failures, as well as health providers' and beneficiaries' perspectives of implementing the strategy in Malawi.

\section{Methods}

As a cross-sectional implementation research, the study was carried out in three southern Malawi districts of Chiradzulu, Mangochi and Zomba. In each district, four health centres and 16 villages were randomly selected to participate. A mixed-methods approach to data collection focusing on quantitative data for coverage and knowledge, attitudes and practices assessments; and qualitative data for assessing perceptions of health providers and beneficiaries regarding MDA was used. Quantitative data were processed and analyzed using IBM SPSS software version 26 while qualitative data were analysed using NVivo 12 for Windows.

\section{Results}

Knowledge levels about schistosomiasis and STH in the districts varied according to disease aspects asked about. Majority are more knowledgeable about what schistosomiasis is (78\%) and whether STH are treatable with drugs $(97 \%)$; with least knowledgeable about the organism that transmits schistosomiasis (18\%), types of schistosomiasis (11\%) and what causes STH (20\%). In 2018 and 2019 the districts registered high coverage rates for praziquantel and albendazole using community-based MDA (73\%-100\%) and using school-based MDA (75\%-91\%). Both the health authorities and community members perceived the MDA approach as good because it brings treatment closer to people.

\section{Conclusion}

With the high MDA coverage obtained in schools and communities, the implementation of MDA in the target districts is satisfactory. There are, however, several challenges including disproportionate knowledge levels, which are hampering progress towards attainment of the 2030 global NTD goals. There is a need for promotion of community participation and partnerships as well as implementation of other recommended interventions for sustainable prevention and control of schistosomiasis and STH.

\section{Background}

Neglected tropical diseases (NTD) represent a diverse group of human and zoonotic diseases whose health and economic burden fall most heavily on the poorest people and communities [1]. These include diseases such as soil transmitted helminthiases (STH), lymphatic filariasis (LF), onchocerciasis, schistosomiasis, dengue and rabies among the 20 listed diseases [1]. Globally, at least 1.7 billion people in 185 countries are affected by NTD and require mass or individual treatment and care to minimize the burden. Of these, 1.1 billion (65\%) are in low- and middle-income countries (LMIC) which are affected by at least five NTD [1]. Access to health interventions continues to be a major challenge for many people in rural settings in LMIC of the world including subSaharan Africa where health systems are weak and suffer from inadequate mechanisms for delivering vital health services to those in most need [2, 3]. Preventive chemotherapy using mass drug administration (MDA), case-management, vector management, environmental improvement measures and health promotion are the key interventions recommended by World Health Organization (WHO) for prevention and control of NTD [4].

In Malawi, most water bodies (20\% of the country) are considered to be potential transmission sites for schistosomiasis [5] and schistosomiasis and STH, are some of the NTD that require special emphasis due to their considerable public health significance. These infections are highly prevalent amongst school aged children as well as adults in most parts of the country. Various surveys carried across the country dating from 1994 to 2018 show that schistosomiasis and STH are major public health problems with prevalence ranges of between $50.0 \%-94.9 \%$ for urinary schistosomiasis caused by Schistosoma haematobium, 34.0\%-67.0\% for intestinal schistosomiasis caused by Schistosoma mansoni and 0.3\%-3.9\% for STH mainly Ascaris lumbricoides (roundworms) and Ancylostoma sp. (hookworms) [5-17]. MDA is one of the community-based interventions, which has been widely carried out annually since 2012 [18]. During annual MDA campaigns, it is the health workers, mostly the community-based Health Surveillance Assistants (HSA) who are responsible for the distribution of drugs. Over the years of MDA implementation in Malawi, community coverage has been high at $87.0 \%$ (range: $51.5 \%-95.0 \%$ ) for praziquantel and $82.0 \%$ (range: $30.6 \%-92.3 \%$ for albendazole due to donor support by the Schistosomiasis Control Initiative Foundation (SCIF) [18]. However these high coverage rates pose a sustainability question for the MDA deliveries once the donor support comes to the end.

Since the start of delivery of MDA in Malawi, no study has been carried out to assess the effectiveness of MDA on prevention and control of schistosomiasis and STH, and to document the perceptions of health providers and beneficiaries regarding MDA for disease prevention and control. The study also looked at MDA coverage, examined perceived strengths and weaknesses, successes and failures, as well as health providers' and beneficiaries' perspectives of implementing the MDA strategy in Malawi in order to draw key lessons to inform programmatic planning for future sustainable schistosomiasis and STH control. 


\section{Methods \\ Study design}

The study was designed as a cross-sectional baseline study for subsequent implementation research to assess coverage, examine perceived strengths and weaknesses, successes and failures, as well as health providers' and beneficiaries' perspectives of implementing the MDA strategy for prevention and control of selected NTD in selected Malawi districts.

\section{Study setting}

Malawi is a landlocked country in South-eastern Africa with a 2020 projected population of 20,119,830 inhabitants [19]. The Malawi Government is the main public provider for health care services providing free health care services at the point of delivery close to $60 \%$ of all health care services through the Ministry of Health $(\mathrm{MOH})$ [20]. The primary health strategy for the $\mathrm{MOH}$ is to ensure attainment of the universal health coverage (UHC) through the implementation of the essential health package (EHP) programme [21]. The EHP programme addresses the major causes of morbidity and mortality among the general population and focuses particularly on medical conditions and service gaps that disproportionately affect the rural poor. The EHP has identified the prevention and control of schistosomiasis and STH as one of its priority areas toward attainment of the UHC. Prevention and control of NTD in Malawi benefits from the existence of a national plan of action for NTD [5] and its incorporation in the overall Ministry of Health strategic plan [20]. Additionally, in the Malawi context disease control takes advantage of current political will, well established partnerships, the occurrence of a defined structure for managing NTD spanning from national to district levels including the presence of skilled HSA and the availability of volunteers in the communities [20,22].

\section{Study areas}

This study was carried out in three southern Malawi districts of Mangochi, Zomba and Chiradzulu (Figure 1). The districts were selected purposively based on their level of co-endemicity with the prioritized NTD of schistosomiasis and STH. Other criteria included districts' distinct topographies with both inland and lowland communities, which may have significant impact on the ecology and consequently on the transmission dynamics and prevalence of schistosomiasis and STH.

These districts are also amongst the high transmission areas with considerable prevalence of infection for both schistosomiasis and STH in Malawi [5-17]. The selection process for study sites involved consultations with the Director of Preventive Health Services and the National Schistosomiasis and STH Control Programme (NSCP) Manager. Selection of health centres and villages involved consulting respective District Health Management Team (DHMT) members in the target districts. From an exhaustive list of functioning health centres located in the three target districts, four health centres and 16 villages per district were randomly selected to participate in the study. Comparative socio-economic, demographic and health indicators for the three study districts and for Malawi as a whole are presented in Table 1.

Table 1

Comparative socio-economic, demographic and health indicators for the three study districts and for Malawi

\begin{tabular}{|c|c|c|c|c|}
\hline Indicator & Chiradzulu & Mangochi & Zomba & Malawi \\
\hline Population size/Density ${ }^{\star}$ & $446,521 / 424$ & $1,177,300 / 168$ & $909,107 / 316$ & $20,119,830 / 186$ \\
\hline Predominant religion $^{*}$ & $\begin{array}{l}\text { Christian } \\
(87.7 \%)\end{array}$ & Islam (70.3\%) & $\begin{array}{l}\text { Christian } \\
(76.8 \%)\end{array}$ & $\begin{array}{l}\text { Christian } \\
(79.9 \%)\end{array}$ \\
\hline Level of literacy among women/men ${ }^{*}$ & $77.8 \% / 93.3 \%$ & $57.0 \% / 71.1 \%$ & $77.9 \% / 87.7 \%$ & $72.1 \% / 82.9 \%$ \\
\hline$\%$ of population with improved sanitation facilities ${ }^{*}$ & $57.0 \%$ & $62.0 \%$ & $60.0 \%$ & $63.0 \%$ \\
\hline Infant mortality rate ${ }^{\star \star}$ & $45 / 1,000$ & $37 / 1,000$ & $80 / 1,000$ & $42 / 1,000$ \\
\hline Fertility rate ${ }^{\star \star}$ & 5.1 & 5.9 & 5.4 & 4.4 \\
\hline Maternal mortality rate ${ }^{\star \star \star}$ & $64 / 100,000$ & $400 / 100,000$ & $210 / 100,000$ & $439 / 100,000$ \\
\hline$\%$ of population with safe water ${ }^{\star \star *}$ & $73.0 \%$ & $74.0 \%$ & $82.0 \%$ & $67.0 \%$ \\
\hline No. of health workers (doctors/nurses/CHW per 100,000 population) ${ }^{+}$ & $0.9 / 30.0 / 46.1$ & $0.4 / 17.9 / 40.9$ & $0.9 / 26.5 / 65.9$ & $1.9 / 33.7 / 76.2$ \\
\hline No. of health facilities per 100,000 population ${ }^{+}$ & 3.6 & 3.6 & 4.1 & 4.7 \\
\hline $\begin{array}{l}\text { Gross Domestic Product \% of population under the poverty line (1US } \\
\text { Dollar) }{ }^{++}\end{array}$ & $65.4 \%$ & $69.8 \%$ & $67.5 \%$ & $65.3 \%$ \\
\hline
\end{tabular}

Sources: *Malawi Census Report, 2018 [19]; ${ }^{* *}$ Malawi Demographic and Health Survey, 2017 [24]; ${ }^{* \star}$ District Social Economic Profiles for Chiradzulu, 2017 [25], Mangochi, 2017 [26] and Zomba, 2017 [27]; ${ }^{+}$Health Sector Strategic Plan II, 2017 [20] \& ${ }^{++}$Malawi Growth and Development Strategy III, 2017 [28].

\section{MDA services, process and delivery}

Mass drug administration is a means of delivering safe and inexpensive medicines based on the principles of preventive chemotherapy, where populations or sub-populations are offered treatment without prior individual diagnosis [4, 29-31]. The NSCP has since 2012 been organizing annual MDA campaigns in 
every district in order to distribute praziquantel and albendazole drugs against schistosomiasis and STH respectively. In every district, the campaigns are implemented by a multi-sectoral team led by NTD and School Health and Nutrition (SHN) Coordinators from the Ministries of Health and Education, Youth and Sports respectively. At community level, distribution of drugs is done by HSA with help from teachers in schools and community volunteers in villages. Supervision and monitoring of MDA delivery are done by health workers from the district and health centres. During these campaigns, it is mainly the school age children (SAC) aged between 5 and 15 who are targeted and prioritized although adults aged above 15 years also receive treatment. Implementation of MDA in Malawi follows the process and guidelines as laid down by WHO [4, 29-31].

\section{Study population and sampling techniques}

The 2020 projection of total population living in the 48 sampled villages was 53,954 of which $52.4 \%$ were female and $47.6 \%$ were male. Of the total population 15,401 (28.6\%) were from Chiradzulu [25], 27,026 (50.2\%) were from Mangochi [26] and 11,444 (21.2\%) were from Zomba [27]. All children aged above 5 years and adults constituted the study population.

Sampling techniques deployed at district level included purposive selection of key informants namely, the District NTD Coordinators, Pharmacy Technicians, and representatives of some of the partners involved in NTD or MDA delivery in each district. At health facility level, it was mostly the officers in-charge (clinicians or nurses) or their representatives and Senior Health Surveillance Assistants who participated in the study. For in-depth interviews (IDI) at community level, we selected community leaders from the targeted villages while taking into consideration their diverse gender and roles. In addition, to obtain a varied community representation and a detailed impression of community perceptions we invited different homogenous groups of eight to ten people from selected villages to participate in a focus group discussion (FGD). Lastly, in every village a predetermined number of households were randomly selected to participate in a questionnaire-based knowledge, attitudes and practices (KAP) survey. Any household member above 14 years available during the time of the visit was invited to participate in the survey while ensuring gender balance.

\section{Data collection}

The study employed a mixed-methods approach to data collection focusing on quantitative data for coverage and qualitative data for assessing perceptions of health providers and beneficiaries and evaluating processes regarding MDA intervention. Data were collected from 2 nd to 17 th April, 2020 at district, health centre and village levels in all study districts. Trained research assistants collected the necessary data for the study from the involved health professionals, implementation partners, community leaders, MDA implementers and beneficiaries using data collection instruments previously used by the research group in 2010 and 2016 studies [23, 32]. The instruments consisted of a survey questionnaire programmed in tablets and was administered to adult household representatives at community level for determining respondents' knowledge, attitudes and practices regarding prevention and control of schistosomiasis and STH, and delivery of MDA. Interview guides were used to conduct in-depth interviews with professionals at district and health centre levels, implementation partners at district level and MDA implementers at community level about their perceptions on benefits, critical factors and to evaluate the processes used during MDA intervention delivery. Moreover, schistosomiasis and STH treatment records for preceding years of 2018 and 2019 were obtained from health services and reviewed using checklists to establish MDA coverage data at district, health centre and village levels. Focus group discussion guides were used to conduct group interviews with beneficiaries about their perceptions on using the MDA intervention and benefits. All the proceedings of the key informant indepth interviews and focus group discussions were recorded using digital audio recorders. Using these tools data were collected in three districts comprising totals of 12 health centres and 48 villages. Finally, document reviews were carried out in order to get an insight on the national prescription of health policy, priority health issues, strategy and effectiveness of delivery of MDA, availability of resources for MDA and the existing challenges and opportunities. Table 2 summarizes the methods, purposes, levels and quantities of data collected in the study.

Table 2

Methods, purposes, levels and amount of data collected in the study

\begin{tabular}{|c|c|c|c|c|c|c|}
\hline \multirow[t]{2}{*}{ Methods } & \multirow[t]{2}{*}{ Purpose of data collected } & \multicolumn{5}{|c|}{ Data collection - levels and numbers collected } \\
\hline & & District & $\begin{array}{l}\text { Implementation } \\
\text { partners }\end{array}$ & $\begin{array}{l}\text { Health } \\
\text { Centre }\end{array}$ & Village & Househc \\
\hline 1. Questionnaire & Knowledge, attitudes and practices & - & - & - & - & 379 \\
\hline 2. In-depth interviews & Process/Perceptions/Benefits/Critical factors & 6 & 3 & 12 & 41 & - \\
\hline $\begin{array}{l}3 . \\
\text { Checklists/Observations }\end{array}$ & Coverage/Disease burden & 6 & - & 12 & - & - \\
\hline $\begin{array}{l}\text { 4. Health Management } \\
\text { and Information System }\end{array}$ & Coverage/Disease burden & 3 & - & - & - & - \\
\hline $\begin{array}{l}\text { 5. Focus group } \\
\text { discussions }\end{array}$ & Perceptions/Benefits/Critical factors & - & - & - & 12 & - \\
\hline 6. Document reviews & Policies/Processes/Priorities/Resources/Challenges/Opportunities & 6 & - & - & - & - \\
\hline
\end{tabular}

\section{Data management and analysis}

Quantitative data collected through survey questionnaire was programmed in tablets using the Secure Data Kit (SDK) formerly LINKS [33]. Questionnaire and checklists data were processed and analyzed using IBM Statistical Package for Social Sciences (SPSS) software version 26 . Analysis involved calculation of percentages, tabulations and frequencies to estimate MDA coverage. Furthermore, statistical significance tests using Chi Square were performed on parameters to measure association between survey respondents' socio-economic state and their level of knowledge in relation to schistosomiasis and STH. 
Qualitative data consisted of textual and audio data, including translated transcripts of key informant in-depth interviews, transcripts of focus group discussions, field notes on observations and other intervention-specific insights, and notes and reports from meetings. Qualitative content analyses of the data was done using NVivo 12 for Windows (QSR International) [34].

\section{Results}

\section{Assessment of knowledge, attitudes and practices regarding NTD and MDA}

The survey on knowledge, attitudes and practices levels regarding NTD and MDA reached a total of 379 respondents comprising 268 (70.7\%) female and 111 (29.3\%) male drawn from the three districts of Chiradzulu, Mangochi and Zomba (Figure 1). The mean age of the respondents was 40.7 years ranged between 16 and 89 years and majority (93.9\%) of them resided in rural areas. Table 3 summarizes distribution of the socio-economic characteristics of respondents across the study districts.

Table 3

Socio-economic characteristics of survey respondents

\begin{tabular}{|c|c|c|c|c|}
\hline \multirow[t]{2}{*}{ Characteristic } & \multicolumn{4}{|c|}{ Number (\%) of respondents who participated in the survey } \\
\hline & Chiradzulu & Mangochi & Zomba & Totals \\
\hline 1. No. of respondents & $126(33.3)$ & $129(34 \%)$ & $124(32.7 \%)$ & $379(100)$ \\
\hline 2. Sex & $91(72.2)$ & $92(71.3)$ & $85(68.5)$ & $268(70.7)$ \\
\hline Female & $35(27.8)$ & $37(28.7)$ & 39 (31.5) & $111(29.3)$ \\
\hline \multicolumn{5}{|l|}{ Male } \\
\hline 3. Age (in years) & 42.5 & 36.9 & 42.6 & 40.7 \\
\hline Mean & $18-89$ & $18-87$ & $16-81$ & $16-89$ \\
\hline \multicolumn{5}{|l|}{ Range } \\
\hline 4. Location & $21(16.7)$ & - & $1(0.8)$ & $23(6.1)$ \\
\hline Urban & $105(83.3)$ & $129(100)$ & $123(99.2)$ & $356(93.9)$ \\
\hline \multicolumn{5}{|l|}{ Rural } \\
\hline 5. Marital status & $5(4)$ & $11(8.6)$ & $4(3.2)$ & $20(5.3)$ \\
\hline Single & $86(68.3)$ & $104(80.6)$ & 101 (81.5) & $291(76.8)$ \\
\hline Married & $18(14.3)$ & $8(6.2)$ & $5(4)$ & $31(8.2)$ \\
\hline Divorced & $13(10.3)$ & $3(2.3)$ & 13 (10.5) & $29(7.6)$ \\
\hline Widowed & $4(3.2)$ & $3(2.3)$ & $1(0.8)$ & $8(2.1)$ \\
\hline \multicolumn{5}{|l|}{ Separated } \\
\hline 6. Education level & $13(10.3)$ & $41(31.8)$ & $20(16.1)$ & 74 (19.5) \\
\hline None & $82(65.1)$ & $67(51.9)$ & $80(64.5)$ & $229(60.4)$ \\
\hline Primary & $30(23.8)$ & $20(15.5)$ & $22(17.8)$ & $72(19)$ \\
\hline Secondary & $1(0.8)$ & $1(0.8)$ & $2(1.6)$ & $4(1.1)$ \\
\hline \multicolumn{5}{|l|}{ Tertiary } \\
\hline 7. Occupation & $34(27)$ & 19 (14.7) & $29(23.4)$ & $82(21.6)$ \\
\hline Business & $72(57.1)$ & $92(71.3)$ & $72(58.1)$ & $236(62.3)$ \\
\hline Farmer & - & $2(1.6)$ & $7(5.6)$ & $9(2.4)$ \\
\hline Fisher & $6(4.8)$ & - & $5(4)$ & $11(2.9)$ \\
\hline Employed & $14(11.1)$ & $11(8.5)$ & $10(8.1)$ & $35(9.2)$ \\
\hline Unemployed & - & $5(3.9)$ & $1(0.8)$ & $6(1.6)$ \\
\hline Other & & & & \\
\hline
\end{tabular}

Knowledge levels about schistosomiasis varied among respondents according to aspects asked about and district. The survey revealed that a majority of the respondents is highly knowledgeable about what schistosomiasis is (78\%). However, respondents' knowledge levels declined when asked to mention what causes schistosomiasis (41\%), intermediate organism for schistosomiasis (18\%) and the types of schistosomiasis (11\%). Among the participating districts Chiradzulu generally fared better in terms of knowledge levels followed by Zomba and Mangochi (Figure 2). 
With regards to knowledge of STH, the survey results (Figure 3) revealed that knowledge levels of STH varied across the study districts depending on the question that was asked. Majority of the respondents were highly knowledgeable about whether STH are treatable with drugs (97\%) and what STH are (50\%). However, respondents had low knowledge levels when asked to mention what causes STH (20\%). Among the districts, Zomba (range: $21 \%-99 \%)$ and Chiradzulu (range: 7\%-99\%) scored better in terms of STH knowledge levels than Mangochi (range: 9\%-91\%).

The survey delved to inquire attitudes of respondents towards community based health service delivery process in relation to MDA. The majority expressed positive views as evidenced when $89.4 \%$ agreed that there very were few health services in the communities, which they said necessitates community members to take some of the health care responsibilities in health services delivery. They were of the view that involvement of community members in health activities will enhance health for people in their community (96.3\%). As regards to community involvement in drug distribution, $93.9 \%$ opined that it helps in saving the time of the health worker to do other things, and that it is a good way to make drugs available to the people ( $95.2 \%)$. However, $65.2 \%$ were of the view that the distribution of drugs like praziquantel and albendazole is best done by health workers, and that communities are not capable of organizing and monitoring treatment of schistosomiasis and intestinal worms on their own (46.4\%). About $68.1 \%$ of the respondents thought that community members should not handle drugs for schistosomiasis and intestinal worms because they are not trained as health workers. Only $8.4 \%$ said that community involvement in schistosomiasis and intestinal worms' treatment is a take-over of the duties of the health worker while majority (85.5\%) agreed that community members are capable of supervising the treatments of schistosomiasis and intestinal worms during MDA.

The survey sought to elucidate the issues surrounding practices related to NTD and MDA by the respondents (Table 4). About a quarter (23.5\%) of the respondents revealed that they had ever suffered from schistosomiasis for which $88.8 \%$ were able to receive treatment. In general about $51.4 \%$ of the respondents had recently received schistosomiasis drugs where Chiradzulu got more (58.7\%) followed by Zomba (53.2\%) and Mangochi (42.6\%). Majority of those who received schistosomiasis drugs got them within their communities (69.7\%) followed by from health facilities (18.5\%) and schools (11.8\%). The drugs were mainly dispensed by HSA (49.7\%), facility-based health workers (45.7\%) and community-based volunteers (4.6\%). Asked if the respondents had experienced any problem after taking the schistosomiasis drugs, $23.6 \%$ answered affirmatively. Majority reported feelings of drowsiness or dizziness (65.2\%), followed by abdominal pains (13\%) and nausea or vomiting (10.9\%). Less than half of the respondents (41.2\%) reported that schistosomiasis drugs are readily accessible by people in villages.

Table 4

Practices related to NTD and MDA of respondents by districts

\begin{tabular}{|c|c|c|c|c|}
\hline \multirow[t]{2}{*}{ Practice issues asked } & \multicolumn{4}{|c|}{ Number of respondents who agreed (\%) } \\
\hline & Chiradzulu & Mangochi & Zomba & Totals \\
\hline \multirow[t]{2}{*}{ a) Have you ever suffered from schistosomiasis? } & $N=126$ & $N=129$ & $N=124$ & $N=379$ \\
\hline & $23(18.2)$ & $36(27.9)$ & $30(24.2)$ & $89(23.5)$ \\
\hline \multirow[t]{2}{*}{ b) If yes, did you get drugs for treatment of schistosomiasis? } & $\mathrm{N}=23$ & $N=36$ & $N=30$ & $\mathrm{~N}=89$ \\
\hline & $20(87)$ & $34(94.4)$ & $25(83.3)$ & $79(88.8)$ \\
\hline \multirow[t]{2}{*}{ c) Have you recently received drugs for schistosomiasis? } & $N=126$ & $N=129$ & $N=124$ & $N=379$ \\
\hline & $74(58.7)$ & $55(42.6)$ & $66(53.2)$ & $195(51.4)$ \\
\hline d) Where did you get the drugs from? & $\mathrm{N}=74$ & $\mathrm{~N}=55$ & $N=66$ & $N=195$ \\
\hline - Community & $58(78.4)$ & $27(49.1)$ & $51(77.3)$ & $136(69.7)$ \\
\hline - Health facility & $14(18.9)$ & $16(29.1)$ & $6(4.1)$ & $36(18.5)$ \\
\hline - School & $2(2.7)$ & $12(21.8)$ & $9(13.6)$ & $23(11.8)$ \\
\hline e) Who dispensed the schistosomiasis drugs to you? & $\mathrm{N}=74$ & $\mathrm{~N}=55$ & $N=66$ & $N=195$ \\
\hline • Facility health worker & $33(44.6)$ & $26(47.3)$ & $30(45.4)$ & $89(45.7)$ \\
\hline - Community health worker & $36(48.6)$ & $27(49.1)$ & $34(51.5)$ & $97(49.7)$ \\
\hline - Community health volunteer & $5(6.8)$ & $2(3.6)$ & $2(3)$ & $9(4.6)$ \\
\hline \multirow[t]{2}{*}{ f) Did you experience any problem(s) after taking schistosomiasis drugs? } & $N=74$ & $N=55$ & $N=66$ & $N=195$ \\
\hline & $11(14.9)$ & $17(30.9)$ & $18(27.3)$ & $46(23.6)$ \\
\hline g) If yes, what problem did you experience after taking schistosomiasis drugs? & $\mathrm{N}=11$ & $\mathrm{~N}=17$ & $\mathrm{~N}=18$ & $N=46$ \\
\hline - Drowsiness/dizziness & $4(36.3)$ & $11(64.6)$ & $15(83.2)$ & $30(65.2)$ \\
\hline - Abdominal pain & $3(27.3)$ & $2(11.8)$ & $1(5.6)$ & $6(13)$ \\
\hline - Nausea/vomiting & $2(18.2)$ & $2(11.8)$ & $1(5.6)$ & $5(10.9)$ \\
\hline - Other & $2(18.2)$ & $2(11.8)$ & $1(5.6)$ & $5(10.9)$ \\
\hline \multirow[t]{2}{*}{ h) Are schistosomiasis drugs readily accessible in this village? } & $N=126$ & $N=129$ & $N=124$ & $N=379$ \\
\hline & $48(38.1)$ & $61(47.3)$ & $47(37.9)$ & $156(41.2)$ \\
\hline
\end{tabular}




\section{Assessment of MDA coverage}

The study assessed MDA coverage targeting delivery of praziquantel and albendazole for schistosomiasis and STH respectively. MDA coverage data for these two NTD for the consecutive years of 2018 and 2019 were obtained from the three study districts. The MDA deliveries in 2018 and 2019 were done by HSA both in schools and communities in all the districts. A comparison of the coverage across the districts during the two years revealed that all the districts registered high coverage rates for praziquantel using community-based MDA (range: 73\%-100\%) and using school-based MDA (range: 75\%-91\%). For community MDA for praziquantel, Chiradzulu district scored highly above the national coverage rates in the two years. As for school-based MDA, Zomba and Chiradzulu districts were the highest scoring above the national rates both in 2018 and 2019 (Figure 4).

Similarly, high coverage trends for community (range: 73\%-90\%) and for schools (range: 75\%-92\%) were observed in albendazole MDA in all the districts for 2018 and 2019. In 2018 and 2019, Chiradzulu and Zomba respectively had highest albendazole coverage using the community MDA delivery approach. As for school coverage, Zomba and Chiradzulu were highest for 2018 and 2019, respectively (Figure 5).

A comparison between albendazole MDA coverage rates for 2018 and 2019 revealed that there were no differences among the districts with regards to praziquantel and albendazole distribution. No differences were also observed for both praziquantel and albendazole between community and school modes of MDA deliveries.

\section{Health authorities and community perceptions on priority health issues and MDA}

The study sought to understand the perceptions on priority health issues and MDA by health authorities as providers and community members as beneficiaries of health services in the three districts. On health issues, both groups agreed on Covid-19, malaria, schistosomiasis, human immunodeficiency virus (HIV) and diarrhoea as health issues requiring priority attention. Some differences between perceptions of health providers and beneficiaries regarding priority health interventions were observed. In addition, the health authorities mentioned acute respiratory infections (ARI), sexually transmitted infections (STI) and tuberculosis (TB) as priority issues. However, communities went further by mentioning inadequate health workers, natural disasters, unclean water, poor sanitation, and lack of amenities such as clinics, ambulances, bicycles, protective wear, drugs, mosquito nets and health education materials as deserving priority attention. These are some of the health determinants or risk factors for the mentioned diseases.

On the community-based delivery of MDA, health authorities perceived the approach as good because it brings treatment closer to people. These sentiments were also shared by community members who exalted the role they play during MDA delivery:

"This is a welcome idea to us communities since we are experiencing shortage of health workers in the area to deliver health services. If a community takes part in the delivery of health services the development of the area will likely to improve." - FGD young male, Chiradzulu

While most community members applauded delivery of MDA, some expressed misgivings because the health system prioritizes children over adults although both schistosomiasis and STH are prevalent in both groups of people in endemic areas.

\section{"[...] it is good but they give more to children ignoring the rest of us." - FGD adult male, Mangochi}

\section{Availability of partners and resources for MDA}

According to key informant interviews, it was learned that although MDA delivery is primarily a responsibility of the MOH through the NSCP, there are a number of partners who assist by providing various support towards implementation of MDA. The support is mostly in form of technical advisory, research, financial, personnel, logistics and supplies which are provided at various levels of health care system. At national level, the partnership for MDA comprise the two Government Ministries for Health and Education, Youth and Sports and various non-governmental organizations who play several roles. At district level there was a presence of partners such as GIZ through the Nutrition and Access to Primary Education (NAPE) project in Chiradzulu, Save the Children in Zomba and the Blantyre Institute of Community Outreach (BICO) in Mangochi who assist the districts by providing various resources such as transport and feeding pupils during implementation of MDA. However, in the study districts, the presence of partner organisations at facility and community levels was scarce (Table 5).

Table 5

Implementation partnerships, resources and roles in MDA

\begin{tabular}{|llll|}
\hline No. & Name of partner & Type & Resources and role played \\
\hline 1. & Ministry of Health & Government & Personnel, logistics and overall implementation \\
\hline 2. & Ministry of Education, Youth and Sports & Government & Personnel and logistics \\
\hline 3. & World Health Organization & UN agency & Technical advisory and supplies \\
\hline 4. & Schistosomiasis Control Initiative Foundation & NGO & Financial and logistics \\
\hline 5. & World Vision International & NGO & Financial and logistics \\
\hline 6. & The German Agency for International Cooperation (GIZ) & NGO & Financial and logistics \\
\hline 7. & Save the Children & NGO & Financial and logistics \\
\hline 8. & Centre for Health, Agriculture Development Research and Consulting (CHAD) & NGO & Financial, research and logistics \\
\hline 9. & Blantyre Institute of Community Outreach (BICO) & NGO & Financial, research and logistics \\
\hline 10. & Research for Health Environment and Development (RHED) & NGO & Research \\
\hline
\end{tabular}

Page $7 / 17$ 


\section{Community participation in MDA delivery}

Although MDA delivery is inherently a community and school based service, it is mostly the health professionals from national and district levels who are responsible for the planning and implementation. At the grassroots level, it is generally the HSA assisted by the teachers and community health volunteers who are involved in MDA delivery in schools and communities respectively. During interviews held with various health authorities and community members it was learned that community involvement and participation are restricted to community sensitization and actual drug distribution. Both health authorities and community members share positive perceptions on the use of community-based modes of delivering health services during MDA delivery. These communitybased approaches enhance accessibility of the services and furthermore, allow community participation in solving their own health problem which will bring a sense of ownership and sustainability of MDA services.

\section{"[...] community participation in health delivery is good, it reduces workload" - Medical Assistant, Chiradzulu}

"Community participation makes the work of health workers easier by supporting in some cases like emergency conditions. It helps to improve the quality of health services like immunization and to promote good hygiene practices." - A community leader, Zomba

\section{Challenges associated with MDA delivery}

The study identified challenges associated with MDA delivery from both the NSCP and involved study districts perspectives. According to the interview with the NSCP manager, the following were some of the challenges at the national level:

a. Existence of research gaps leading to limited information on available current efforts regarding effective options for prevention and control of NTD.

b. Non-existent vector control activities as one of the five integrated public health interventions recommended by WHO for prevention and control of NTD specifically through transmission control.

c. Inadequate linkages with the national WASH programme also in the MOH despite existence of evidence that joint efforts between NTD and WASH can lead to simpler, more cost-effective and streamlined interventions.

d. Inadequate support by private sector and other partners in prevention and control of NTD where it is mostly a few non-governmental organizations who are involved as implementation partners.

e. Disproportionate level of knowledge of schistosomiasis and STH diseases which retards efforts towards implementation of effective MDA delivery for long term prevention, control and elimination of NTD.

Similarly, during interviews held with NTD coordinators and health workers the following were identified as challenges incurred during delivery of MDA in the study districts:

a. Lack of partners to support MDA delivery at district and facility levels for prevention and control of NTD.

b. Apart from health education, no other complementary interventions such as WASH and vector control are implemented during MDA campaigns.

c. Scheduling of annual MDA campaigns by the NSCP mostly coincide with the rainy season when people are busy with agricultural activities.

d. In some instances poor community engagement strategies have led to people not embracing MDA services.

\section{Discussion}

This paper has presented an assessment of MDA implementation for prevention and control of NTD, in particular schistosomiasis and STH in three southern Malawi districts. The findings of the assessment have revealed that the three study districts consistently achieved high MDA coverage rates in 2018 and 2019 for both schistosomiasis and STH drugs. This achievement is possible due to the collaboration through the NSCP and health care structures in the respective districts together with partners and community members. The assessment has shown that the MDA as a strategy is an effective approach for availing the much needed NTD drugs to people in the involved districts. These findings agree with similar studies carried out elsewhere [35]. There was no observed differences in coverage rates between MDA delivered in communities and schools; also between praziquantel and albendazole. However, despite the high coverage rates obtained in the study districts over the two years there is only slow progress towards achievement of the goal to reduce burden of schistosomiasis and STH to levels of no public health importance in Malawi by 2025. Recent prevalence studies done across Malawi after introduction of MDA in 2012, including in the study districts have pointed to persistent infections [6, 13-17, 37-42] and reinfections [37-42] occurring within a year despite sustained annual MDA campaigns implemented throughout the country. These persistent infections and reinfections are similar to those observed in other studies carried out in Cote d'Ivoire, Kenya, Mozambique, Tanzania [43] and Togo [44]. In the wake of these revelations, there is a need to reevaluate the current WHO guidelines that recommend that MDA delivery every 12 months in areas categorized as low-to-medium transmission settings [44-47]. Another possible factor affecting the progression towards elimination of these diseases is lack of a coordinated implementation of other interventions along with MDA for effective prevention and control of schistosomiasis and STH. These other interventions are intensified disease management to reduce morbidity, vector and ecology management for transmission control, public health services and provision of safe water, sanitation and hygiene [3,4,29, 34, 48, 49].

The need to engage the public in NTD prevention and control activities has become imperative in the context of morbidity reduction through MDA delivery and community participation [50]. An increase in the awareness of community members regarding schistosomiasis and STH is important for them to play a central role in the prevention and control of these diseases [51]. This study has found that knowledge levels about schistosomiasis and STH varied disproportionately between survey respondents according to aspects asked about and district. This study has shown that although many respondents have heard about schistosomiasis and STH prevention and control activities, their knowledge on specific aspects of the diseases is low relative to the percentage of 
the respondents that knowledge about the diseases. This disparity could be due to poor packaging of messages during delivery of health education for schistosomiasis and STH. These finding are similar to studies done in Nigeria, Egypt, Cameroon, Papua New Guinea, Turkey and Philippines which demonstrated that despite implementation of numerous activities towards the control of NTDs, there is little sensitization of the general public in order to increase awareness of the diseases [50-55].

The assessment has found that during MDA delivery, community involvement and participation are restricted to community sensitization and actual drug distribution times. There is a need for more involvement and participation during MDA planning and implementation in order to enhance the spirit of ownership and empowerment. Furthermore, it was learned that there are a number of partners who assist the Government through the MOH by providing support towards implementation of various MDA activities. Availability of partners, resources and public-private partnerships are vital in prevention and control programmes to ensure that the progress made towards schistosomiasis and STH prevention and control in Malawi is consolidated and sustained [34, 56-58]. The 2017 - 2022 Malawi Health Sector Strategic Plan II provides a rallying point for various health partners to participate in health delivery endeavours [20], the implementation of MDA also requires availability of partners and resources for its effective delivery [35].

Perceptions about diseases are important factors in shaping the necessary policies toward the prevention and control of such diseases. In the context of schistosomiasis and STH, perceptions and public knowledge may influence community's decision-making for drug uptake and policy formulation for MDA implementation. In this assessment, both health authorities and community members perceived the community-based approach of MDA delivery as good because it brings treatment closer to people. However, some community members expressed a concern that the health system prioritizes children over adults although both schistosomiasis and STH are prevalent between both groups of people in endemic areas. These perceptions are similar to those expressed in other studies carried out in Turkey, Ghana and Tanzania [54, 55, 59].

This paper has documented several challenges associated with implementation of MDA for prevention and control of schistosomiasis and STH according to the NSCP and districts. Despite the many challenges faced by the health sector in implementation of MDA, the authorities remain committed to improving accessibility to community health services including MDA for schistosomiasis and STH prevention and control. These challenges are similar to those encountered in most Asian and Sub-Sahara African countries as raised in a review by Bergquist and Gray (2019) [60]. In order to tackle the challenges associated with implementation of MDA towards the elimination of NTD, there is a need for embracing other WHO recommended interventions and community-based health initiatives by actively engaging communities themselves.

Our study has some limitations; the data were collected from only three districts from the southern region of Malawi. Although it was not the intention from the outset that it should represent the whole country, the interviews with national authorities and reviews of official documents suggest that our findings could be generalized. The inclusion of highly endemic districts and use of multiple data sources are the major strengths of this study. As possible areas of future research, we would recommend inclusion of prevalence and cost-effectiveness assessments of MDA delivery to enrich the findings of similar studies, and also to test the effects of implementation of several interventions such as vector control, WASH, enviromental management, using an integrated approach in a longitudinal study in at least one of the districts with high prevalence of these NTD.

\section{Conclusions}

Our findings indicate that the implementation of MDA in the three target districts is satisfactory. The districts have been registering high coverage for praziquantel and albendazole both in schools and communities. However, persistent infection and reinfection rates recorded within a year after delivery of MDA are casting a doubt on the prospects of attaining the set goal of reducing the burden of schistosomiasis and STH to levels of no public health importance by 2025. This study has identified several operational challenges including lack of vital NTD related knowledge by the public which are hampering efforts by the NSCP and partners towards the global NTD goals by 2030. There is an urgent need for promotion of community participation and partnerships as well as a coordinated implementation of other interventions along with MDA for effective prevention and control of schistosomiasis and STH.

\section{List Of Abbreviations}

ARI Acute Respiratory Infections

BICO Blantyre Institute of Community Outreach

CHAD Centre for Health, Agriculture Development Research and Consulting

CHAM Christian Health Association of Malawi

DHMT District Health Management Team

EHP Essential Health Package

FGD Focus Group Discussion

GIZ Die Deutsche Gesellschaft für Internationale Zusammenarbeit (The German Agency for International Cooperation)

HIV Human Immunodeficiency Virus

HSA Health Surveillance Assistant 


\begin{tabular}{|c|c|}
\hline HSSP & Health Sector Strategic Plan \\
\hline IDI & In-depth Interview \\
\hline KAP & Knowledge, Attitudes and Practices \\
\hline LMIC & Low and Medium Income Countries \\
\hline $\mathrm{MOH}$ & Ministry of Health \\
\hline NAPE & Nutrition and Access to Primary Education \\
\hline NGO & Non-Governmental Organization \\
\hline NHSRC & National Health Sciences Research Committee \\
\hline NSCP & National Schistosomiasis and Soil-transmitted Helminths Control Programme \\
\hline MDA & Mass Drug Administration \\
\hline NTD & Neglected Tropical Diseases \\
\hline RHED & Research for Health Environment and Development \\
\hline SDG & Sustainable Development Goal \\
\hline SDK & Secure Data Kit \\
\hline SHN & School Health and Nutrition \\
\hline SPSS & Statistical Package for Social Sciences \\
\hline STH & Soil-Transmitted Helminths \\
\hline STI & Sexually Transmitted Infections \\
\hline TB & Tuberculosis \\
\hline $\mathrm{UHC}$ & Universal Health Coverage \\
\hline UN & United Nations \\
\hline WHO & World Health Organization \\
\hline
\end{tabular}

\section{Declarations}

\section{Ethics and consent to participate}

In this study, all methods were carried out in accordance with relevant guidelines and regulations. The study protocol, including the informed consent forms were reviewed and approved by the Malawi National Health Sciences Research Committee (NHSRC) (Approval \#2443) prior to the commencement of the study. Informed consent was individually obtained from every participant at the very beginning before proceeding with their involvement after being explained to clearly about the study objectives and aims using local languages of Chichewa and Chiyao used in the study areas. For participants who were literate, a written consent was obtained while for those illiterate a written informed consent to participate was obtained through their legally authorized representatives (literate family members) instead. Similarly for the study participants who were children (under 16 years of age), consent to participate was collected from their parents or guardians on their behalf. All the research instruments that were used at community level were also translated to local languages.

\section{Consent for publication}

Not applicable. The manuscript does not contain any individual person's data in any form.

\section{Availability of data and materials}

The datasets used and/or analyzed during the current study are available from the corresponding author on reasonable request.

\section{Competing interests}

The authors declare that they have no competing (financial and non-financial) interests. 


\section{Funding}

This work received financial support from the Coalition for Operational Research on Neglected Tropical Diseases (COR-NTD), which is funded at The Task Force for Global Health primarily by the Bill \& Melinda Gates Foundation, by the Foreign, Commonwealth and Development Office of the British government, and by the United States Agency for International Development through its Neglected Tropical Diseases Program (REF: NTD-SC\#195D). These funding organizations did not play any role in the design of the study, collection, analysis and interpretation of data, and in writing the manuscript. The authors of this paper alone are responsible for the views expressed in this publication which do not necessarily represent the decisions or policies of the Coalition for Operational Research on Neglected Tropical Diseases.

\section{Authors' contributions}

PM and PF conceived the project. PM, SAK, KCM, GB, MF, JM, LTJ and PF designed the study and the tools, conducted selection and preparation of study areas, trained and supervised data collectors. PM, SAK, KCM, GB, MF, JM, LTJ and PF analyzed and interpreted the data. All authors participated in writing and approved the final manuscript.

\section{Acknowledgements}

The authors would like to acknowledge the Director of Preventive Health Services in Ministry of Health, Dr Storn Kabuluzi, Directors of Health and Social Services and staff of the District Health Offices, health centres and implementation partners in Chiradzulu, Mangochi and Zomba district councils for their involvement in this study. The study would not have been possible without the contribution of community members who willingly participated and provided information. The authors are grateful to Mr Innocent Mvula, Mr Noel Zondola (deceased), Mr Daniel Njoka-Mwanza, Mr Austin Zgambo, Mr Moses Ngwira, Mr Mustapha Maulidi and Mr Mwai Chipeta for their support rendered during the implementation of the study.

\section{References}

1. World Health Organization. Working to overcome the global impact of Neglected Tropical Diseases. First WHO Report on Neglected Tropical Diseases. Geneva, update 2011; https://apps.who.int/iris/handle/10665/44778.

2. Bright T, Felix L, Kuper H, Polack S. A systematic review of strategies to increase access to health services among children in low and middle income countries. BMC Health Serv Res. 2017; 17:252 DOI 10.1186/s12913-017-2180-9.

3. Leech AA, Kim DD, Cohen JT, Neumann PJ. Are low and middle-income countries prioritising high-value healthcare interventions? BMJ Glob Health. 2020; 5:e001850. doi:10.1136/ bmjgh-2019-001850.

4. World Health Organization. Sustaining the drive to overcome the global impact of Neglected Tropical Diseases. Second WHO report on Neglected Tropical Diseases. Geneva, 2013; http://www.who.int/iris/bitstream/10665/77950/1/9789241564540_eng.pdf.

5. Malawi Government. Ministry of Health Master Plan for Neglected Tropical Diseases (NTD) for Malawi 2015-2020.

6. Mtethiwa AH, Nkwengulila G, Bakuza J, Sikawa D, Kazembe A: Extent of morbidity associated with schistosomiasis infection in Malawi: a review paper. Infect Dis Poverty. 2015; 4:25. https://doi.org/10.1186/s40249-015-0053-1.

7. Alharbi MH, Condemine C, Christiansen R, LaCourse EJ, Makaula P, Stanton MC, et al. Biomphalaria pfeifferi snails and intestinal schistosomiasis, Lake Malawi, Africa, 2017-2018. Emerg Infect Dis. 2019; https://doi.org/103201/eid2503.181601.

8. Makaula P, Sadalaki JR, Muula AS, Kayuni S, Jemu SK, Bloch P: Schistosomiasis in Malawi: a systematic review. Parasit Vectors. $2014 ; 7: 570$. Doi:10.1186/s13071-014-0570-y

9. Phiri K, Whitty CJM, Graham SM, Ssembatya-lule G: Urban/rural differences in prevalence and risk factors for intestinal helminth infection in southern Malawi. Annals Trop Med Parasitol. 2000; 94(4):381-387. Doi:10.1080/00034983.2000.11813553.

10. Bowie C, Purcell B, Shaba B, Makaula P, Perez M: A national survey of the prevalence of schistosomiasis and soil transmitted helminths in Malawi. BMC Inf Dis. 2004; 4:49-57. https://doi.org/10.1186/1471-2334-4-49.

11. Msyamboza K, Ngwira B, Banda R, Nkwanda S, Brabin B: Sentinel surveillance of Lymphatic filariasis, Schistosomiasis, Soil transmitted helminths and Malaria in rural southern Malawi. Malawi Med J. 2010; 22(1):12-14.

12. Madsen H, Bloch P, Makaula P, Phiri H, Furu P, Stauffer Jr JR: Schistosomiasis in Lake Malawi villages. EcoHealth. 2011; 8:163-176. https://doi.org10.1007/s10393-011-0687-9.

13. Kayuni S, Peeling R, Makaula P: Prevalence and distribution of Schistosoma haematobium infection among school children living in southwestern shores of Lake Malawi. Malawi Med J. 2017; 29(1):16-23. Doi:10.4314/mmj.v29i1.4.

14. Pullanikkatil D, Mubako S, Phalira W, Chiotha S, Luhanga M: Schistosomiasis prevalence in Zomba, Southern Malawi. Afr Geogr Rev. 2014; 33(1):36-51. https://doi.org/10.1080/19376812.2013.861758.

15. Mtethiwa AHN, Bakuza J, Nkwengulila G: Prevalence and intensity of Schistosomiasis in communities around water reservoirs in Malawi. J Trop Dis. 2015; 4:183. doi: 10.4172/2329-891X.1000183.

16. Chipeta MG, Ngwira B, Kazembe LN: Analysis of Schistosomiasis haematobium infection, prevalence and intensity in Chikhwawa, Malawi: an application of a Two Part Model. PLoS Negl Trop Dis. 2013; 7(3):e2131. Doi:101371/journal.pntd.0002131. 
17. Poole H, Terlouw DJ, Naunje A, Mzembe K, Stanton M, Betson M, Lalloo DG, Stothard JR: Schistosomiasis in pre-school-age children and their mothers in Chikhwawa district, Malawi with notes on characterization of schistosomes and snails. Parasit Vectors. 2014; 7:153-164. https://doi.org/10.1186/17563305-7-153.

18. Ministry of Health and Population, National Schistosomiasis and STH Control Programme. Report of 2018 MDA Campaign. Lilongwe, Malawi.

19. National Statistical Office. The 2018 Malawi Census Report, 2019. Zomba, Malawi.

20. Republic of Malawi, Health Sector Strategic Plan II 2017 - 2022 Towards Universal Health Coverage. Ministry of Health and Population, Department of Planning 2017. Lilongwe, Malawi.

21. Republic of Malawi, A joint programme of work for a health sector wide approach (SWAp) 2004-2010. Ministry of Health and Population, Department of Planning 2004. Lilongwe, Malawi.

22. Republic of Malawi, National Community Health Strategy 2017 - 2022 integrating health services and engaging communities for the next generation. Ministry of Health and Population, Department of Planning 2017. Lilongwe, Malawi.

23. Makaula P, Bloch P, Banda H, Mbera GB, Mangani C, de Sousa A, Nkhono E, Jemu S: Primary Health Care in rural Malawi - a qualitative assessment exploring the relevance of the community-directed interventions approach. BMC Health Serv Res. 2012; 12:328. https://doi.org/10.1186/1472-6963-12328.

24. National Statistical Office. Malawi Demographic and Health Survey, 2017. Zomba, Malawi.

25. Chiradzulu District Council. District Social Economic Profile for Chiradzulu, Malawi, 2017.

26. Mangochi District Council. District Social Economic Profile for Mangochi, Malawi, 2017.

27. Zomba District Council. District Social Economic Profiles for Zomba, Malawi, 2017.

28. Republic of Malawi. Ministry of Economic Planning and Development. Malawi Growth and Development Strategy III, 2017. Lilongwe, Malawi.

29. World Health Organization. Preventive chemotherapy in human helminthiasis. Coordinated use of anthelminthic drugs in control interventions: a manual for health professionals and programme managers. Geneva, 2006. https://apps.who.int./iris/handle/10665/43545.

30. Montresor A, Crompton DWT, Gyorkos TW, Savioli L. Helminth control in school age children: a guide for managers of control programmes. World Health Organization. Geneva, 2016. https://www.who.int/neglected_diseases/resources/9789241548267/en/.

31. Montresor A, Mupfasoni D, Mikhailov A, Mwinzi P, Lucianez A, Jamsheed M, et al. The global progress of soil-transmitted helminthiases control in 2020 and World Health Organization targets for 2030. PLoS Negl Trop Dis. 2020; 14(8), p.e0008505. doi:101371/journal.pntd.0008505.

32. Makaula P, Funsanani M, Mamba KC, Musaya J, Bloch P: Strengthening Primary Health Care at district-level in Malawi - determining the coverage, costs and benefits of Community-Directed Interventions. BMC Health Serv Res. 2019; 19<bi>:</bi>509 https://doi.org/10.1186/s12913-019-4341-5.

33. Pavluck A, Chu B, Flueckiger RM, Ottesen E: Electronic Data Capture Tools for Global Health Programs: Evolution of LINKS, an Android-, Web-Based System. PLoS Negl Trop Dis. 2014; 8(4): e2654. doi:10.1371/journal.pntd.0002654.

34. Vaismoradi M, Turunen $\mathrm{H}$, Bondas T. Content analysis and thematic analysis: Implications for conducting a qualitative descriptive study. Nurs Health Sci. 2013; 15(3): 398-405. Doi:10.1111/nhs.12048. Epub 2013 Mar 11. PMD: 23480423.

35. Engels D and Zhou X. Neglected tropical diseases: an effective global response to local poverty-related disease priorities. Infect Dis Poverty. 2020; 9:10 https://doi.org/10.1186/s40249-020-0630-9.

36. Binder S, Campbell CH, Castleman JD, Kittur N, Kinung'hi SM, Olsen A, et al. Lessons Learned in Conducting Mass Drug Administration for Schistosomiasis Control and Measuring Coverage in an Operational Research Setting. Am J Trop Med Hyg. 2020; 103 (Suppl 1) pp. 105-113 doi.10.4269/ajtmh.19-0789.

37. Kayuni SA, LaCourse EJ, Makaula P, Lampiao F, Juziwelo L, Fawcett J, et al. Case Report: Highlighting Male Genital Schistosomiasis (MGS) in Fishermen from the Southwestern Shoreline of Lake Malawi, Mangochi District. Am J Trop Med. Hyg. 2019; pp. 1-5. https://doi:10.4269/ajtmh.19-0562.

38. Kayuni SA, Corstjens PLAM, LaCourse EJ, Bartletti KE, Fawcett J, Shaw A, Makaula P et al. How can schistosome circulating antigen assays be best applied for diagnosing male genital schistosomiasis (MGS): An appraisal using exemplar MGS cases from a longitudinal cohort study among fishermen on the south shoreline of Lake Malawi. Parasitology. 2019; https://doi.org/10.1017/S0031182019000969.

39. Webster BL, Alharbi MH, Kayuni S, Makaula P, Halstead F, Christiansen R et al. Schistosome Interactions within the Schistosoma haematobium Group, Malawi. Emerg Infect Dis. 2019; DOI: https://doi.org/10.3201/eid2506.190020.

40. Stothard JR, Kayuni SA, Al-Harbi MH, Musaya J, Webster BL. Future schistosome hybridizations: Will all Schistosoma haematobium hybrids please standup! PLoS Negl Trop Dis. 2020; 14(7): e0008201. https://doi.org/10.1371/journal. pntd.0008201.

41. Kayuni SA, Angus OM, Baxter H, Hesketh J, Mainga B, Lally Jr D et al. An outbreak of intestinal schistosomiasis, alongside increasing urogenital schistosomiasis prevalence, in primary school children on the shoreline of Lake Malawi, Mangochi District, Malawi. Infect Dis Poverty. $2020 ; 9: 121$. https//doi.org/10.1186/s40249-020-00736-w.

42. Kayuni SA, Alharbi MH, Makaula P, Lampiao F, Juziwelo L, La Course EJ, Stothard JR. Male Genital Schistosomiasis Along the Shoreline of Lake Malawi: Baseline Prevalence and Associated Knowledge, Attitudes and Practices Among Local Fishermen in Mangochi District, Malawi. Public Health Front. 2020; 9:590695. doi: 10.3389/fpubh.2021.590695.

43. Kittur N, King CH, Campbell Jr. CH, Kinung'hi S, Mwinzi PNM, Karanja DMS et al. Persistent Hot spots in Schistosomiasis Consortium for Operational Research and Evaluation Studies for Gaining and Sustaining Control of Schistosomiasis after Four Years of Mass Drug Administration of Praziquantel. Am J Trop Med Hyg. 2019; pp. 1-11 doi:10.4269/ajtmh.19-0193.

44. Bronzan RN, Dorkenoo AM, Agbo YM, Halatoko W, Layibo Y, Adjeloh P et al. Impact of community-based integrated mass drug administration on schistosomiasis and soil-transmitted helminth prevalence in Togo. PLoS Negl Trop Dis. 2018; 12(8): e0006551. https://doi.org/

Page $12 / 17$ 
10.1371/journal.pntd.0006551.

45. Anderson R, Truscott J, Hollingsworth TD. The coverage and frequency of mass drug administration required to eliminate persistent transmission of soiltransmitted helminths. Phil Trans R Soc. 2014; B 369: 20130435. http://dx.doi.org/10.1098/rstb.2013.0435.

46. Friedman JF. Optimizing Delivery of Mass Drug Administration for Schistosomiasis. Am J Trop Med Hyg. 2019; 101(6): 1191-1192 doi:10.4269/ajtmh.19-0762.

47. Kura K, Hardwick RJ, Truscott JE, Toor J, T. Hollingsworth TD, Anderson RM. The impact of mass drug administration on Schistosoma haematobium infection: what is required to achieve morbidity control and elimination? Parasit Vectors. 2020; 13:554 https://doi.org/10.1186/s13071-020-04409-3.

48. Ackley C, Elsheikh M, Zaman S. Scoping review of Neglected Tropical Disease Interventions and Health Promotion: A framework for successful NTD interventions as evidenced by the literature. PLoS Negl Trop Dis. 2021; 15(7): e0009278. https://doi.org/10.1371/journal. pntd.0009278.

49. Ross AGP, Olveda RM, Chy D, Olveda DU, Li Y, Harn DA et al. Can Mass Drug Administration Lead to the Sustainable Control of Schistosomiasis? J Infect Dis. 2014; 211. DOI: 10.1093/infdis/jiu416

50. Olamiju OJ, Olamiju FO, Adeniran AA, Mba IC, Ukwunna CC, Okoronkwo C et al. Public Awareness and Knowledge of Neglected Tropical Diseases (NTDs) Control Activities in Abuja, Nigeria. PLoS Negl Trop Dis. 2014; 8(9): e3209. doi:10.1371/journal.pntd.0003209.

51. Elfar E, Asem N, Yousof H. The awareness of neglected tropical diseases in a sample of medical and nursing students in Cairo University, Egypt: A crosssectional study. PLoS Negl Trop Dis. 2020; 14(11): e0008826. https://doi.org/10.1371/journal.pntd.0008826.

52. Kamga HLF, Assob NJC, Nsagha DS, Njunda AL, Njimoh DL. A community survey on the knowledge of neglected tropical diseases in Cameroon. Int J Med Biomed Res. 2012; 1(2):131-140. http://dx.doi.org/10.14194/ijmbr.128.

53. Karoke W. Neglected tropical diseases: less known subjects amongst health care professionals. J Community Med Public Health. $2018 ; 34$ (6): $254-255$.

54. Ulukanligil M. Community perception of school-based deworming program in Sanliurfa, Turkey. Am J Trop Med Hyg. 2006; 75:1063-1068.

55. Inobaya MT, Chau TN, Ng S, MacDougall C, Olveda RM, Tallo VL et al. Mass drug administration and the sustainable control of schistosomiasis: an evaluation of treatment compliance in the rural Philippines. Parasit Vectors. 2018; 11:441 https://doi.org/10.1186/s13071-018-3022-2.

56. Caines K. Global Health Partnerships and Neglected Diseases Part of the 2004 DFID Study: Global Health Partnerships: Assessing the Impact. DFID Health Resource Centre 27 Old Street London EC1V 9HL.

57. Bush S, Hopkins AD. Public-private partnerships in neglected tropical disease control: The role of nongovernmental organisations. Acta Trop. 2011; 120S:S169-S172.

58. Ndayishimiye O, Ortu G, Soares MRJ, Clements A, Willems J, Whitton J et al. Control of Neglected Tropical Diseases in Burundi: Partnerships, Achievements, Challenges, and Lessons Learned after Four Years of Programme Implementation. PLoS Negl Trop Dis. $2014 ;$ 8(5): e2684. http://dx.doi:10.1371/journal.pntd.0002684.

59. Brooker S, Marriot H, Hall A, Adjei S, Allan E, Maier C et al. Community perception of school-based delivery of anthelmintics in Ghana and Tanzania. Trop Med Int Health. 2001; 6:1075-1083

60. Bergquist R, Gray DJ. Schistosomiasis Elimination: Beginning of the End or a Continued March of a Trodden Path? Trop Med Infect Dis. $2019 ; 4: 9$. http://dx.doi:10.3390/tropicalmed4020076.

\section{Figures}




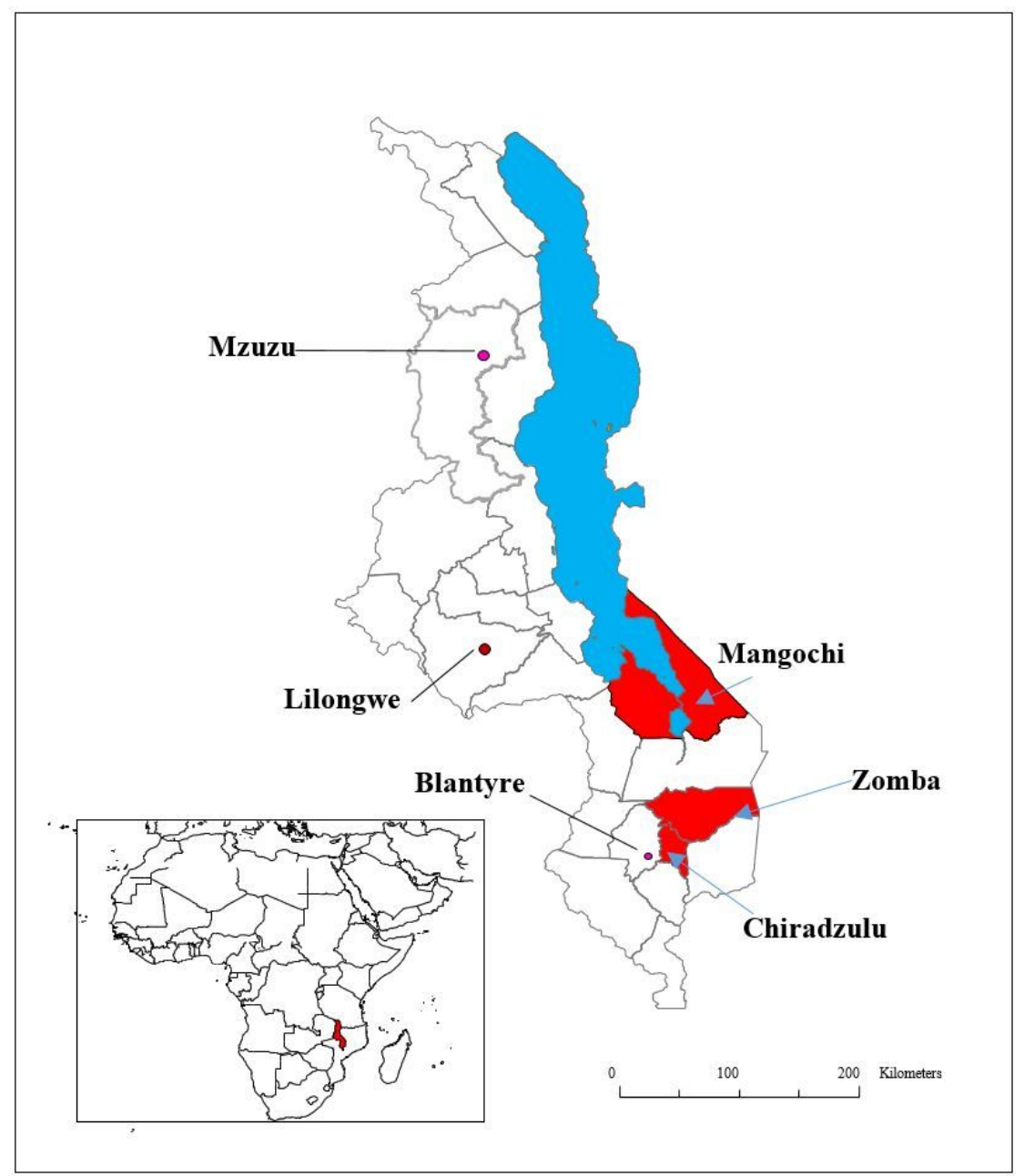

Figure 1

Locations of the study districts of Mangochi, Zomba and Chiradzulu (in red), Lake Malawi (in blue), major cities of Mzuzu, Lilongwe and Blantyre and the location of Malawi in Africa (red in the inset) (Source: Authors' own map [23]) 


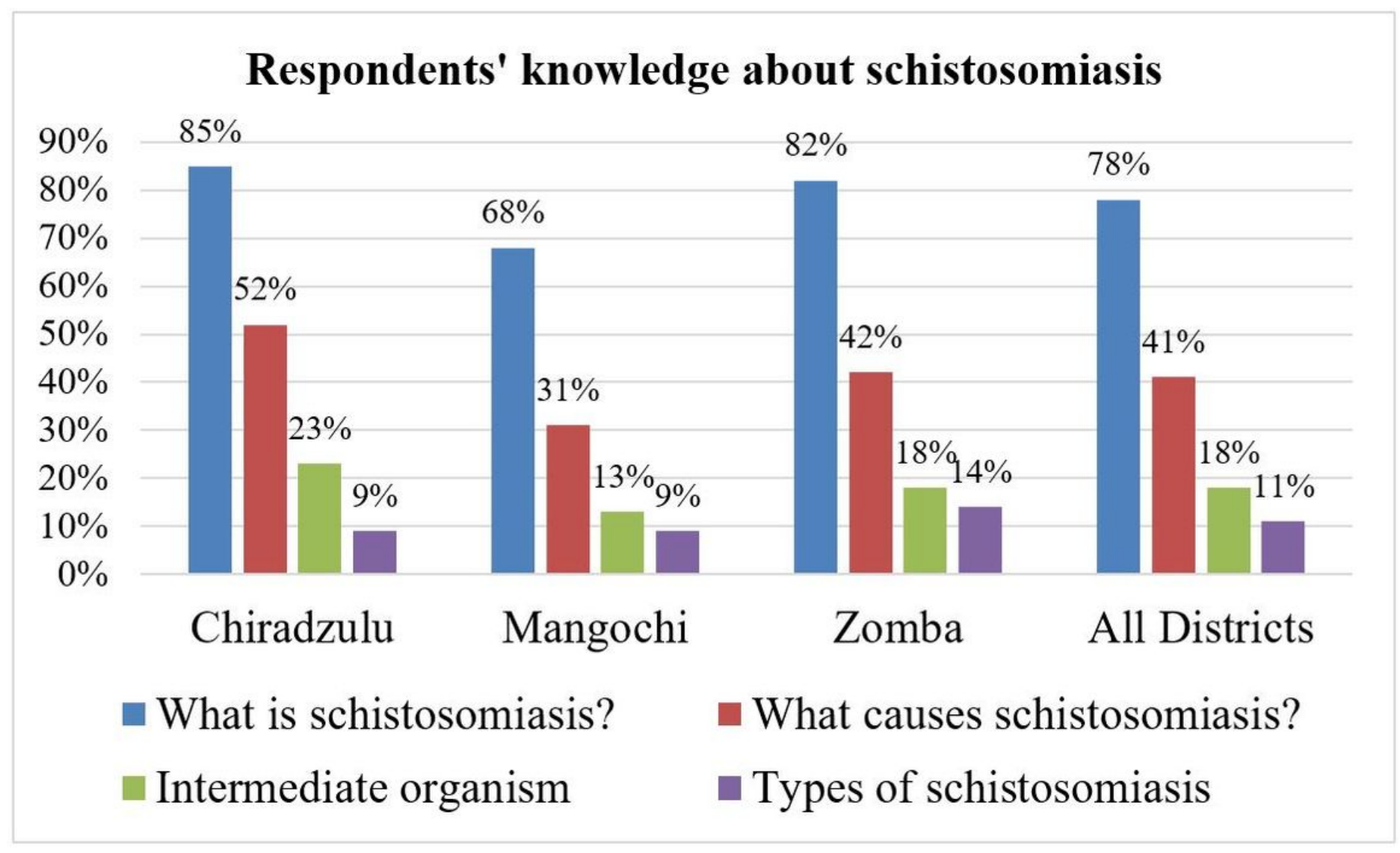

Figure 2

Respondents' knowledge about schistosomiasis 


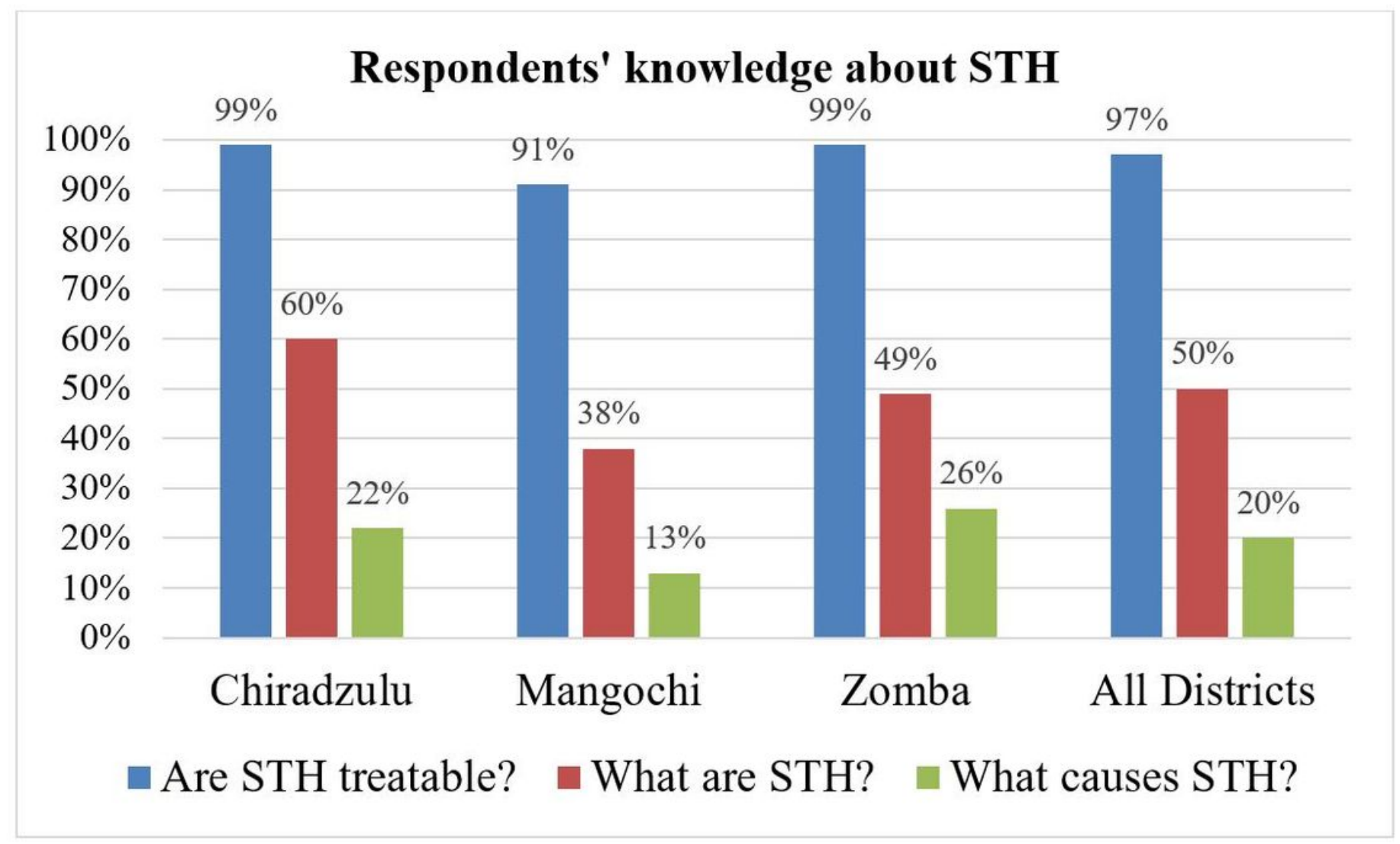

Figure 3

Respondents' knowledge about STH 


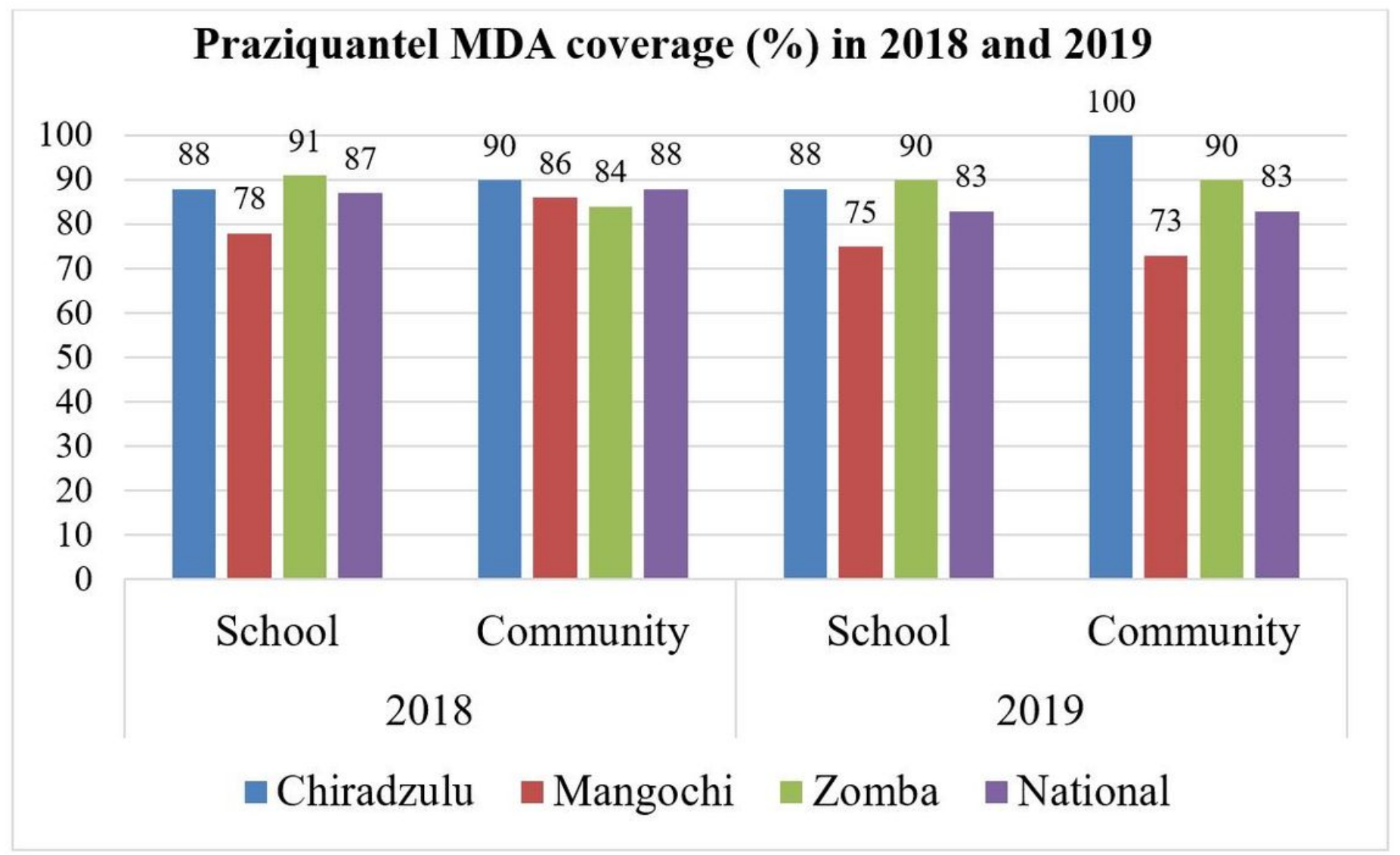

Figure 4

Praziquantel MDA coverage (\%) in years 2018 and 2019 